Ann. Abeille, Ig66, 9 (3), 237-242.

\title{
INFLUENCE DES VARIATIONS DE L'AGE DE MATURITÉ SEXUELLE CHEZ LES REINES D'ABEILLES (APIS MELLIFICA MELLIFICA) FÉCONDÉES PAR INSÉMINATION ARTIFICIELLE
}

\author{
J. FRESNAYE \\ Station expérimentale d'Apiculture, \\ Centre de Recherches agronomiques du Sud-Est, 84-Montfavet
}

SOMMAIRE

Les résultats d'expériences concernant l'influence de l'âge de puberté des reines sur le pourcentage de reines correctement fécondées par insémination artificielle sont décrits dans cette étude. L'époque de l'élevage des reines et les conditions météorologiques influent sur la durée de l'évolution et sur l'âge de maturité sexuelle de ces reines. Celui-ci est atteint plus rapidement en pleine saison. Il convient donc de respecter des délais variables avant de pratiquer l'insémination artificielle : Io à $\mathrm{I} 2$ jours après la naissance présumée des reines en début de saison, 5 à 6 jours en pleine saison d'élevage.

\section{INTRODUCTION}

Les variations du temps d'évolution et de l'âge de maturité sexuelle sont très fréquentes chez les insectes. Elles sont causées principalement par les différences de température ainsi que par les saisons et peuvent atteindre plusieurs mois dans certains cas. Ces variations sont beaucoup plus faibles chez l'abeille (Apis mellifica L.), insecte social, capable d'assurer une auto-régulation de la température et de la maintenir au niveau nécessaire à la survie de la colonie. Les variations du temps d'évolution ont été étudiées, en ce qui concerne l'abeille, par de nombreux auteurs, et JAY (I963) a fait une revue détaillée et une mise au point de tous ces travaux. Cette analyse montre que le temps de développement entre la ponte et la naissance est de I6 à 24 jours pour les ouvrières, de 20 à 28 jours pour les mâles et de $I_{4}$ à $I_{7}$ jours pour les reines. Outre la température et la saison, la composition et la quantité de nourriture, 
1'âge et la race des nourrices influent également sur le temps d'évolution ainsi que sur le poids de l'abeille ou de la reine à la naissance. Soose (I954) a constaté des variations entre des abeilles de races et de souches différentes et Gon'TARski (I953) signale des arrêts de croissance, qui allongent le temps de cette dernière. MonTAGNER (I962) retrouve ces différences de temps d'évolution chez les reines d'abeille noire française. Il élève des reines à partir de larves de même âge, moins d'un jour, et obtient des naissances pendant 3 jours dans une série, pendant 6 jours dans une autre.

Dans les conditions naturelles d'élevage et de fécondation des reines, les variations de la durée de croissance et de l'âge de maturité sexuelle ne présentent pas d'inconvénient notable. Les reines font leur vol de fécondation lorsqu'elles sont physiologiquement aptes à le faire et dès que les conditions météorologiques le permettent. I1 n'en est pas de même dans le cas de l'insémination artificielle. C'est alors l'expérimentateur qui décide du jour de la fécondation en fonction de la date présımée de la puberté des reines. Car aucun signe extérieur ne permet de déterminer ce moment optimum très variable. Il est influencé par deux variations successives : la durée de croissance avant la naissance qui, généralement, ne peut être constatée au moment précis ou elle se produit, surtout dans les conditions naturelles, suivie de la durée d'évolution, variable elle aussi, avant de parvenir à la maturité sexuelle. Les seules indications morphologiques d'âge des reines au moment de la fécondation sont les modifications du tube digestif et des tubes de Malpighi ainsi que celles de la glande et de la vesicule à venin (FYG, I956; HAYDAK, I957), qui ne sont visibles qu'à la dissection donc inutilisables lorsque l'on veut garder les reines vivantes.

Les premières tentatives de vols de fécondation pourraient être une indication précieuse de la maturité sexuelle des reines. Mais cette méthode imposerait la présence de nombreux observateurs si l'on voulait faire les observations de visu; d'autre part l'utilisation de cages spécialement conçues et fixées à l'entrée des ruchettes de fécondation risquerait de provoquer la blessure ou la mort de nombreuses reines. Il semble que cette méthode soit difficilement praticable.

D'après MACKENSEN et RoBERTs (I948) l'âge le plus favorable à l'insémination artificielle se situe entre le $4^{\mathrm{e}}$ et le Io $^{\mathrm{e}}$ jours après la naissance. Tous les essais effectués avec des reines plus jeunes n'ont donné que de très faibles pourcentages de réussite. Par contre, les reines peuvent encore être inséminées après Io jours et tant qu'elles n'ont pas commencé à pondre parthénogénétiquement. La ponte parthénogénétique d'une reine n'implique d'ailleurs pas une impossibilité de fécondation artificielle ainsi que l'ont démontré CRow et ROBER'TS (I950). Mais à 1'âge approximatif de Io jours apparaît le risque, augmenté ensuite tous les jours, de l'attaque de la reine par les ouvrières qui l'obligent à sortir de la ruche pour être fécondée, l'instinct de reproduction n'étant pas inné mais induit par les ouvrières (RUTTNER I957).

L'âge de fécondation des reines dans les conditions naturelles permet d'estimer l'âge moyen de la puberté et montre encore sa grande variabilité. Soczeck (I958), signale que les reines volent 6 à I3 jours après leur naissance et MEDVEDEv (I956), indique que les reines fécondées commencent à pondre de 7 à $I 9$ jours après l'éclosion. Triasko (I95I), utilisant une technique de fécondation naturelle dans de très petits nuclei de fécondation ne laisse sortir les reines que lorsqu'elles ont atteint l'âge de Io à I2 jours. 
Au cours de plusieurs années de pratique de l'insémination artificielle, il nous est apparu que le pourcentage de réussite pouvait être influencé par les variations du temps de développement et de l'âge de maturité sexuelle des reines. Mais de nombreux autres facteurs influent également sur les résultats. Nous avons rappelé les principaux ainsi que les progrès récents de la technique de l'insémination artificielle dans une revue (FRESNAYE, Ig66) nous n'y reviendrons donc pas.

\section{INFLUENCE DE I'AGE DES REINES SUR IES RÉSULTATS}

\section{DE L'INSÉMINATION ARTIFICIELI,E}

L,es différents facteurs décrits par ailleurs, blessures, infections, volume trop important de sperme, attaque de la reine par les abeilles, sont fréquemment les causes initiales de la mortalité des reines inséminées. Cependant, les résultats obtenus, en suivant les directives données par divers auteurs et spécialistes, et concernant l'âge des reines lors de l'insémination, étaient en début de saison souvent très inférieurs à ceux acquis pendant les mois de juin et juillet. L'évolution de ces variations au cours de la période annuelle d'insémination artificielle nous a amené à supposer que certains aspects de la physiologie des reines pouvaient être incriminés, notamment le temps d'évolution de la reine avant la naissance, étudié par de nombreux auteurs cités plus haut. Ajouté à l'âge de maturité sexuelle, variable également, le délai entre le début de l'élevage de la reine et le moment optimum pour la fécondation peut varier dans des proportions importantes.

Nous présentons les résultats d'une étude faite dans le but de vérifier cette hypothèse. Le tableau I réunit les différents types d'essais effectués pendant les mois les plus favorables à l'élevage et à la fécondation des reines dans notre région : avril, mai, juin et juillet. Au cours de ces différentes périodes de l'année 200 reines environ furent élevées artificiellement par la méthode de surgreffage des larves. Puis elles furent inséminées artificiellement après répartition en deux groupes : les reines inséminées à 1'âge de 5-6 jours d'une part, les reines inséminées à l'âge de Io-I 2 jours d'autre part, en prenant pour base une durée d'évolution moyenne de i 6 jours entre la ponte de l'œuf et l'imago. Les moyennes générales des résultats des différents essais qui donnent 87,I7 p. Ioo de reines fécondes pour des inséminations à IO-I2 jours et $57,60 \mathrm{p}$. I 00 seulement pour les inséminations à $5-6$ jours semblent démontrer une très nette supériorité du premier type de ces essais. Cependant, les résultats partiels indiquent une évolution très nette des pourcentages de reines fécondes. En ce qui concerne le type d'insémination à 5-6 jours, le pourcentage s'améliore progressivement au cours de la saison apicole pour atteindre Ioo p. Ioo au mois de juillet. Par contre, les résultats du type d'insémination à IO-I2 jours sont excellents dès le début de l'expérience et subissent une variation beaucoup moins importante que les précédents, elle est probablement due d'ailleurs à certains des facteurs de mortalité précédemment décrits qui sont extrêmement difficiles à diagnostiquer ainsi qu'à éliminer totalement. La figure I résume cette évolution aboutissant à Ioo p. Ioo de reines fécondes dans les deux types d'insémination au mois de juillet. Cependant, un autre aspect de nos expériences n'apparaît pas dans le tableau I, nous l'avons fait figurer dans le diagramme de la figure I. Nous n'avons en effet indiqué dans le tableau que le nombre 


\section{TABLEAU I}

Résultats des essais d'insémination artificielle de reines âgées de 5 à 6 jours et de 10 à 12 jours pendant 4 mois

\begin{tabular}{|c|c|c|c|c|c|c|}
\hline \multicolumn{2}{|c|}{ Type de l'essai } & \multirow{2}{*}{$\begin{array}{c}\text { Avril } \\
12\end{array}$} & \multirow{2}{*}{$\begin{array}{l}\text { Mai } \\
18\end{array}$} & \multirow{2}{*}{$\frac{\text { Juin }}{11}$} & \multirow{2}{*}{$\begin{array}{c}\text { Juillet } \\
12\end{array}$} & \multirow{2}{*}{$\begin{array}{c}\begin{array}{c}\text { Résultats } \\
\text { globaux }\end{array} \\
53\end{array}$} \\
\hline \multirow{3}{*}{$\begin{array}{l}\text { Reines âgées de } \\
5 \text { à } 6 \text { jours }\end{array}$} & $\mathrm{F}$ & & & & & \\
\hline & $n$ & 27 & 35 & 18 & 12 & 92 \\
\hline & $\%$ & 44,44 & 51,42 & 61,11 & 100,00 & 57,60 \\
\hline \multirow{3}{*}{$\begin{array}{l}\text { Reines âgées de } \\
10 \text { à } 12 \text { jours }\end{array}$} & $\mathrm{F}$ & 11 & 12 & 34 & 11 & 68 \\
\hline & $n$ & 11 & 15 & 41 & 11 & 78 \\
\hline & $\%$ & 100,00 & 80,00 & 82,92 & 100,00 & 87,17 \\
\hline \multirow{3}{*}{ Totaux mensuels } & $\mathrm{F}$ & 23 & 30 & 45 & 23 & 121 \\
\hline & $n$ & 38 & 50 & 59 & 23 & 170 \\
\hline & $\%$ & 60,52 & 60,00 & 76,27 & 100,00 & 71,17 \\
\hline
\end{tabular}

F. nombre de reines fécondes; $n$ nombre de reines inséminées

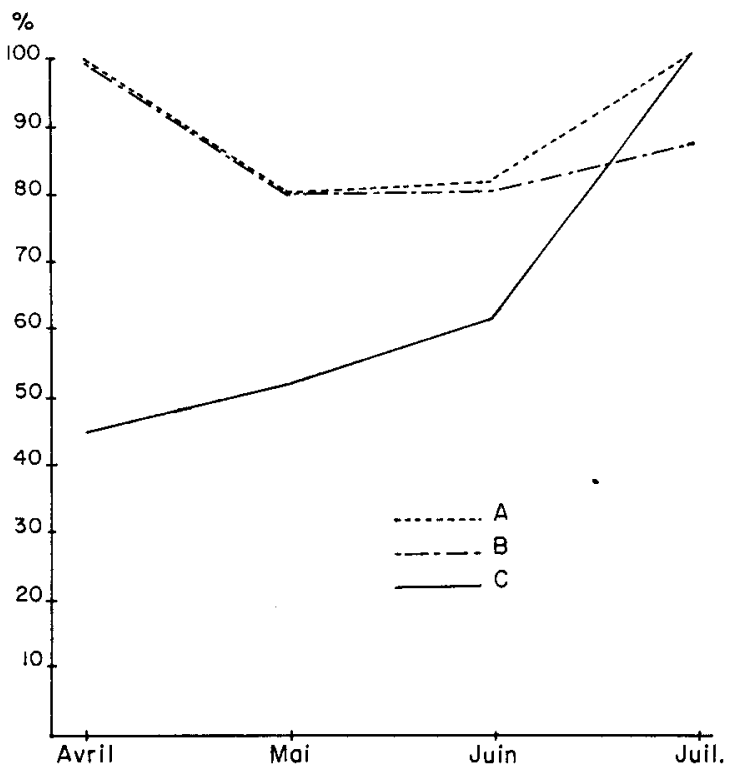

FIG. I. - Pourcentage de reines normalement fécondées, en fonction de l'âge et de la saison

A. Reines âgées de ro à 12 jours (en considérant le nombre de reines inséminées),

B. Reines âgées de 1o à 12 jours (en considérant le nombre de reines avant l'expérience),

C. Reines âgées de 5 à 6 jours. 
de reines inséminées et fécondes de chacun des essais, sans préciser le nombre total de reines avant le début de l'expérience, c'est-à-dire l'ensemble des reines destinées à l'expérience et présentes dans les nuclei. Or, ce nombre, qui reste le même que celui des reines inséminées en ce qui concerne les mois d'avril et mai, est plus important pendant les mois suivants dans les séries d'essais de reines inséminées à l'âge de IO-I 2 jours. Le pourcentage de perte est faible, moins de $5 \mathrm{p}$. IOo des reines en moyenne, pendant le mois de juin. Il atteint environ Io à 15 p. Ioo en moyenne au mois de juillet. Ces reines sont mortes car elles n'ont pas été fécondées alors qu'elles avaient atteint leur maturité depuis plusieurs jours. Plusieurs causes ont pu aboutir à ce résultat: les reines se sont blessées le long des grilles qui les empêchent de sortir, elles ont été attaquées par les abeilles qui tentaient de provoquer le vol de fécondation (RUTTNER, I957) enfin certaines ont pu sortir et n'ont pas pu traverser à nouveau la grille pour rentrer dans le nucléus de fécondation car certaines reines, vierges et de petite taille, arrivent parfois à franchir les grilles d'entrée.

Les résultats de nos expériences montrent que l'insémination artificielle des reines doit être pratiquée à des âges différents suivant la période de l'année, afin d'augmenter les chances de succès. Cet âge varie de Io à I2 jours après la naissance en début de la saison d'élevage, à 5-6 jours pendant les mois de la pleine saison, juin et juillet en ce qui concerne l'abeille noire. Il est possible qu'il existe des différences raciales mais nous n'avons pas encore abordé ce sujet. Les conditions météorologiques pendant l'élevage et la maturation des reines influent vraisemblablement en accélérant ou en retardant l'évolution de celles-ci par rapport à la saison considérée.

\section{Reçu pour publication en août 1966.}

\section{SUMMARY}

THE EFFECT OF VARIATIONS IN THE AGE OF SEXUAL MATURITY OF QUEEN BEES ( "APIS MELLIFICA MELLIFICA ")

FERTILIZED BY ARTIFICIAL INSEMINATION

The results are described of an experiment concerning the effect of the age at which queens reach puberty on the percentage of queens correctly fertilized by artificial insemination. The breeding date of the queen and the meteorological conditions influence the rate of development and the age at which the queens reach sexual maturity. The latter is attained more quickly in high breeding season than at the start of the season. It is therefore expedient to delay before using artificial insemination - Io to 12 days after presumed birth of queens at the beginning of the season, 5 to 6 days in high breeding season.

\section{RÉFÉRENCES BIBLIOGRAPHIQUES}

Crow J. F., Roberts W. C., 1950. Inbreeding and homozygosis in bees. Genetics, 35, 61 2-621.

Fresnaye J., 1964. La claustration des mâles destinés à l'insémination artificielle des reines d'abeilles. Ann. Abeille, 7 , (I), 55 -6r.

Fresnaye J., 1966. L'insémination artificielle des reines d'abeilles. Revue. Ann. Abeille. 9 (3), 25 I-263. FYG W., 1956. Merkmale zur Altersbestimmung von Bienenköniginnen. Schweiz. Bienenzgt., 79, 428-433 Gontarski H., 1953. Zur Brutbiologie der IIonigbiene. Z. Bienenforsch., 2, 7-10. 
HAYDAK H. H., I957. Changes with age in the appearance of some internal organs of the honey-bee. Bee world, 38, $197^{-207}$.

JAy C, r963. The development of honeybees in their cells. J. Apicult. Res., 2, (2), II 7-I34.

Mackensen O., Roberts W. C., 1948. A Manual for the artificial insemination of queen-bees.? U.S.D.A.; Bur. Ent. Planl Quar. E. T., 250, 33 pages.

Medvedev I. K., I956. Sur les conditions qui déterminent le début de la ponte chez la reine d'abeille (en russe). Pschelovodstvo, no II, I0-13.

Montagner H., ig62. Infuence de la technique du double greffage sur le développement des reines d'Apis mellifica. Insecles soc., 9, (1), 91-99.

RutTNer F., I957. Die Sexualfunktion der Honigbienen im Dienste Ihrer sozialen Gemeinschaft. $Z$. vergleich. Physiol., 39, 577-60o.

Soczeck S., I958. Influence of some factors in queens'flights and matings (en polonais). Pszcz. Zesz. Nauk, (2), IO7-I 20 .

Soose E., I954. Einfluss der Temperatur auf die Augsgestaltung von Flugelindex und Panzerfarbe der Honigbiene (Apis mellifica) . Arch. Bienenkde, 31, (2), 49-66.

Triasko V. V., 195I. Méthode de fécondation des reines dans de petits nuclei (en russe). Pschelovodstvo, (9), I 8-25. 